\title{
Absolute Orbit and Gravity Determination using Relative Position Measurements Between Two Satellites
}

\author{
Mark L. Psiaki* \\ Cornell University, Ithaca, N.Y. 14853-7501
}

\begin{abstract}
A method has been developed to use a time series of relative position measurements to estimate the orbits of two satellites along with corrections to the gravity model of the celestial body about which they orbit. This system provides autonomy or near autonomy and could be used for gravity recovery at the Moon or a planet. This system eliminates the need for a third spacecraft that maintains a constant link with Earth-based tracking stations when the two probe spacecraft pass to the far side of the body whose gravity model is being estimated. The system uses a radio cross link to measure the relative range between the two satellites. It uses an optical imaging device on one of the satellites, such as a star camera, and a light beacon on the other satellite in order to measure the relative bearing between the satellites referenced to inertial coordinates. The two satellites' orbits and corrections to the gravity field model are shown to be absolutely observable in cases where the two orbital altitude time histories are not identical. An extended Kalman filter for this system is developed, and it is tested using data from a truth-model simulation. The resulting system can achieve absolute RMS position accuracies of $3.5 \mathrm{~m}$, absolute RMS velocity accuracies of $0.003 \mathrm{~m} / \mathrm{s}$, and RMS gravity accuracies of $3 \times 10^{-7} \mathrm{~m} / \mathrm{s}^{2}$ when operating on a month's worth of data from a pair of Lunar orbiting spacecraft.
\end{abstract}

\section{Introduction}

$\mathrm{T}$ he field of orbit determination is as old as Kepler and has reached a high state of maturity. A modern treatment of orbit determination is contained in a text book that has been written by recognized leaders in the field ${ }^{1}$. Another subject that has a long history is that of gravity model determination based on observations of satellite orbits $^{2,3}$. These techniques use orbit models and measurements in a batch filter or an extended Kalman filter (EKF) to estimate orbit, and if desired, corrections to the gravitational model. Measurements might include GPS pseudorange and Doppler shift, two-way range from a laser ranging system or a satellite-borne radar altimeter, and Doppler shift or integrated Doppler shift from satellite or ground beacons ${ }^{1}$.

The recent initiative to revisit the Moon and eventually explore Mars has generated a renewed interested in developing improved orbit determination methods for satellites that orbit these bodies. This interest, in turn, has generated an interest in the generation of improved gravity models for these bodies because improved gravity models enable increased orbit determination accuracy or a reduced need for measurements in order to achieve a given level of accuracy. The Japanese mission SELenological and ENgineering Explorer (SELENE) is scheduled to launch in 2007 and plans to use inter-satellite Doppler shift measurements between 3 Lunar-orbiting satellites in conjunction with Very Long Baseline Interferometry using Earth-based tracking assets in order to recover a gravity model on the far side of the Moon ${ }^{4}$. The planned Lunar Reconnaissance Orbiter mission is slated to carry a laser altimeter that may be used for simultaneous orbit determination and gravity model estimation ${ }^{5}$. The Gravity Recovery And Interior Laboratory (GRAIL) mission, which is in the concept study phase, also seeks to map the Moon's gravity field, though for scientific rather than operational reasons ${ }^{6}$.

One difficulty of gravity/orbit determination systems for orbits about the Moon or about a planet is their lack of autonomy. Near-Earth systems, such as the Gravity Recovery And Climate Experiment (GRACE) mission, require data from external systems such as the Global Positioning System (GPS) or satellite laser ranging systems. External measurements are not readily available when operating around the Moon or a planet. GPS is unusable, and radio-

\footnotetext{
* Professor, Sibley School of Mechanical and Aerospace Engineering, 206 Upson Hall. Associate Fellow, AIAA.
} 
based navigation must rely on expensive systems such as the Deep-Space Network ${ }^{7}$. The SELENE concept needs to use Earth-based tracking assets in order to carry out its mission ${ }^{4}$, and the GRAIL mission is likely to require similar data. Lunar gravity determination is especially difficult because Earth-based systems cannot track a satellite on the never-visible far side of the Moon. Therefore, missions such as SELENE need to include an extra satellite that is visible both to the Earth and to a second satellite or pair of satellites behind the Moon. The LRO mission's laser altimeter may offer some degree of autonomy for purposes of orbit and gravity model recovery, but it remains unclear how well such a scheme will work.

The present paper seeks to build on an autonomous orbit determination concept that has been proposed for a pair of satellites ${ }^{8,9}$. It determines the absolute orbits of the two satellites based on a time history of measurements of the relative position vector between the two spacecraft, as depicted in Fig. 1. The measurement $\Delta \boldsymbol{r}_{12}\left(t_{k}\right)$ in the figure is the full 3-dimensional position vector of Satellite 1 measured relative to Satellite 2 and referenced to inertial coordinates. The system measures $\Delta \boldsymbol{r}_{12}\left(t_{k}\right)$ by measuring the range between the satellites along with the inertial bearing of Satellite 1 as viewed from Satellite 2. Except for a few special cases that have identical altitude time histories, the absolute orbits of both satellites are observable from a time sequence of relative position measurements $\Delta \boldsymbol{r}_{12}\left(t_{k}\right), \Delta \boldsymbol{r}_{12}\left(t_{k+1}\right), \Delta \boldsymbol{r}_{12}\left(t_{k+2}\right), \ldots{ }^{8}$, and absolute position accuracies on the order of $1 \mathrm{~m}$ may be achievable if a sufficiently accurate measurement system and a sufficiently accurate gravity model are available ${ }^{9}$.

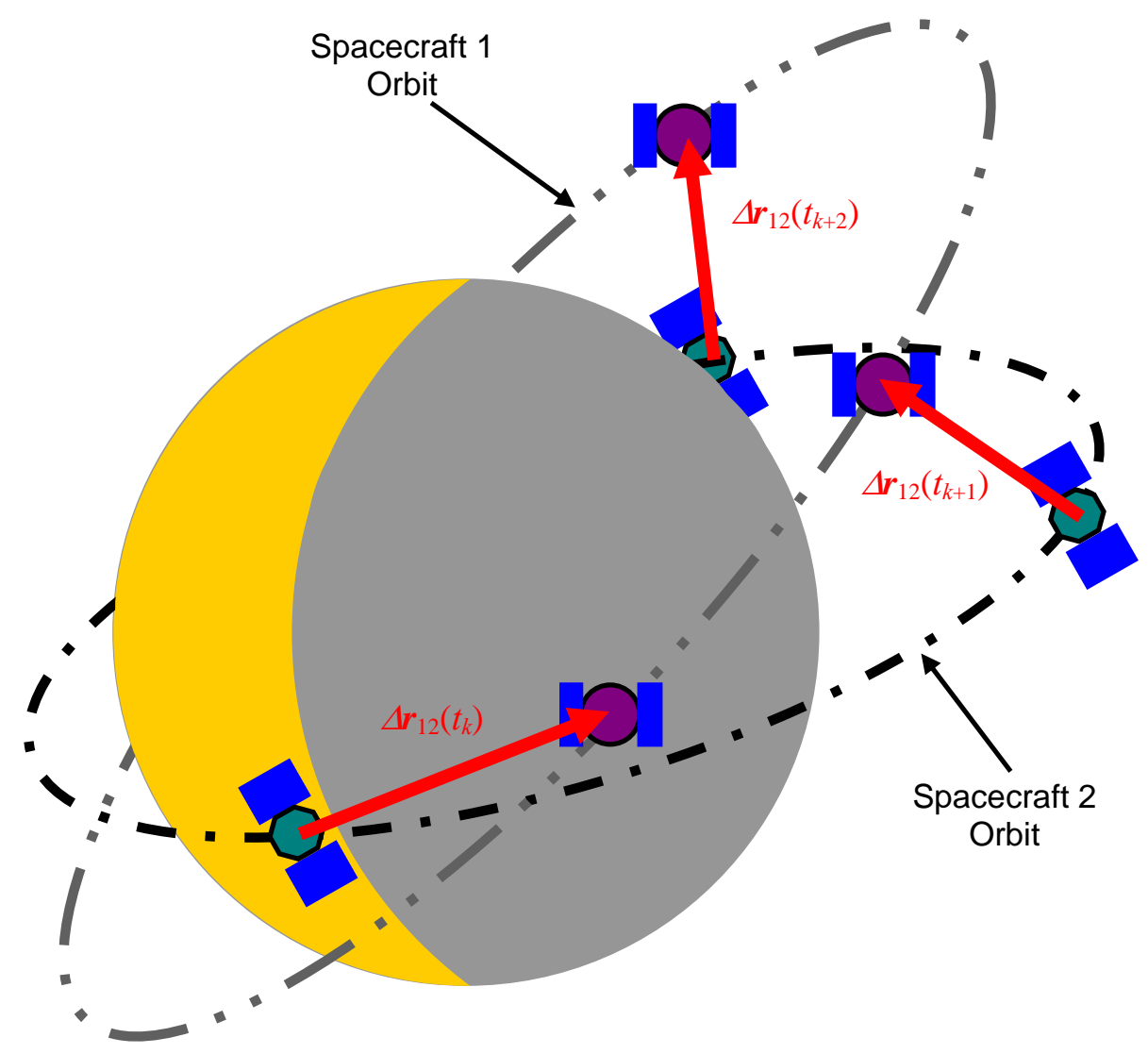

Figure 1. Three samples of the time history of the relative position vector between two Lunar-orbiting satellites.

The goal of the present paper is to investigate whether the system of ${ }^{8,9}$ can be used to estimate corrections to the central body's gravity model in addition to the two satellites' orbits. If workable, then this system would enable autonomous or nearly autonomous GRACE-mission-type determination of gravity models at the Moon, Mars, or other planets. Like GRACE, it would use a radio ranging signal to measure the inter-satellite range. The addition 
of an optical system for measuring the bearing of the $\Delta \boldsymbol{r}_{12}$ vector would make up for the loss of external aids such as GPS or a ground-based laser ranging system. Such a system would be simpler than what is planned for the SELENE mission because it could estimate gravity corrections on the far side of the Moon without the need to have an auxiliary spacecraft that was simultaneously visible from an Earth-based tracking network and from the satellites on the far side of the Moon.

This paper makes four contributions to the study of a dual-spacecraft orbit/gravity estimation system. First, it develops a semi-analytical demonstration of the simultaneous observability of the two orbits and the $1 / r^{2}$ gravitational constant $\mu$ for a simple central-force gravitational field. Second, it sets up a general model and develops an EKF for estimating the following quantities: the positions and velocities of the two satellites, a perturbation to $\mu$, perturbations to the higher-order coefficients in a spherical harmonic expansion of the body's gravitational field, solar radiation pressure coefficients for the two spacecraft, Markov acceleration errors, and sensor biases. The Kalman filter estimates these quantities based on realistic measurements of absolute range, accumulated delta range, and absolute bearing from one spacecraft to the other. Third, this paper performs a linearized numerical observability calculation for its Kalman filter model, and it demonstrates the simultaneous observability of all model states. Fourth, it presents truth-model simulation results that illustrate how this system could perform if applied to a Lunar gravity estimation mission.

The remainder of this paper presents its analyses, models, simulations, and results in five sections plus conclusions. Section II develops a linearized observability analysis for the two spacecraft orbits and the $\mu$ central force parameter of a $1 / r^{2}$ gravity model. Section III presents a system model that includes higher-order gravity terms, non-gravity disturbance accelerations, and realistic measurement models for relative range and bearing. Section IV develops an EKF for the system model of Section III using the Square-Root Information Filter (SRIF) implementation. It also explains how to modify the SRIF calculations in order to numerically compute this system's linearized observability Gramian. Section V describes the truth-model simulation that has been used to study this system's observability and accuracy. Section VI presents the results of the linearized observability calculation along with Kalman filter estimation results for a truth-model simulation of a Lunar gravity recovery mission. Section VII summarizes the paper and presents its conclusions.

\section{Orbit/Gravity Observability in a $1 / \mathbf{r}^{2}$ Central-Force Field}

This section develops a simple orbit/gravity observability analysis for a dual-spacecraft problem with a simple $1 / r^{2}$ central force. This analysis is an extension of that presented in the appendix of ${ }^{8}$. Its goal is to verify that the central gravitational force parameter $\mu$ can be observed simultaneously with the orbits based only on measurements of a time sequence of relative position vectors. A second goal of this analysis is to determine any special orbital cases that are unobservable so that such cases can be avoided in the more complicated numerical calculations of this paper's later sections.

\section{A. Linearized Dynamics Model and Observability Matrix}

Following the approach of ${ }^{8}$, but with modifications to include gravity estimation and some changes of notation, the observability analysis for the central-force version of this system considers the following time-varying linearized system:

$$
\begin{aligned}
& \delta \boldsymbol{x}=A(t) \delta \boldsymbol{x}=\left[\begin{array}{ccccc}
0 & I & 0 & 0 & 0 \\
G_{1}(t) & 0 & 0 & 0 & \gamma_{1}(t) \\
0 & 0 & 0 & I & 0 \\
0 & 0 & G_{2}(t) & 0 & \gamma_{2}(t) \\
0 & 0 & 0 & 0 & 0
\end{array}\right] \delta \boldsymbol{x} \quad \text { with } \delta \boldsymbol{x}=\left[\begin{array}{c}
\delta \boldsymbol{r}_{1} \\
\delta \boldsymbol{v}_{1} \\
\delta \boldsymbol{r}_{2} \\
\delta \boldsymbol{v}_{2} \\
\delta \mu
\end{array}\right] \\
& \delta \boldsymbol{y}=\delta \Delta \boldsymbol{r}_{12}=C \delta \boldsymbol{x}=\left[\begin{array}{lllll}
I & 0 & -I & 0
\end{array}\right] \delta \mathbf{x}
\end{aligned}
$$

where $\delta \boldsymbol{r}_{1}$ and $\delta \boldsymbol{r}_{2}$ are the linearized perturbations to the position vectors of the two spacecraft, $\boldsymbol{r}_{1}$ and $\boldsymbol{r}_{2}, \delta \boldsymbol{v}_{1}$ and $\delta \boldsymbol{v}_{2}$ are the linearized perturbations to the velocity vectors of the two spacecraft, $\boldsymbol{v}_{1}$ and $\boldsymbol{v}_{2}, \delta \mu$ is the linearized perturbation to the attracting body's central force gravitational constant, and $\delta \boldsymbol{y}=\delta \Delta \boldsymbol{r}_{12}$ is the linearized perturbation to the measured relative position vector between the two spacecraft. The 13-by-13 $A(t)$ matrix and the 3-by-13 matrix $C$ are implicitly defined by Eqs. (1a) and (1b). The two matrices 


$$
G_{i}(t)=\frac{\mu}{\left\|\boldsymbol{r}_{i}(t)\right\|^{3}}\left[3 \hat{\boldsymbol{r}}_{i}(t) \hat{\boldsymbol{r}}_{i}^{\mathrm{T}}(t)-I\right] \text { for } i=1,2 \text { with } \hat{\boldsymbol{r}}_{i}(t)=\frac{\boldsymbol{r}_{i}(t)}{\left\|\boldsymbol{r}_{i}(t)\right\|}
$$

are the gravity gradient matrices at the two spacecraft locations, and the vectors

$$
\gamma_{i}(t)=\frac{-\boldsymbol{r}_{i}(t)}{\left\|\boldsymbol{r}_{i}(t)\right\|^{3}} \quad \text { for } i=1,2
$$

equal the nominal gravitational accelerations at the two spacecraft divided by $\mu$. Equations (1a) and (1b) are linearization versions of the dual 2-body problem's original nonlinear model:

$$
\begin{aligned}
& {\left[\begin{array}{c}
\dot{\boldsymbol{r}}_{i} \\
\dot{\boldsymbol{v}}_{i}
\end{array}\right]=\left[\begin{array}{c}
\boldsymbol{v}_{i} \\
-\mu \boldsymbol{r}_{i} /\left\|\boldsymbol{r}_{i}\right\|^{3}
\end{array}\right] \text { for } i=1,2} \\
& \boldsymbol{y}=\Delta \boldsymbol{r}_{12}=\boldsymbol{r}_{1}-\boldsymbol{r}_{2}
\end{aligned}
$$

Note that all position and velocity vectors are defined with respect to an inertially-oriented coordinate system whose origin lies at the center of mass of the attracting central body.

The observability analysis for the time-varying linear system in Eqs. (1a)-(1b) starts by deriving a formula for the measurement and its time derivatives:

$$
\left[\begin{array}{c}
\delta \boldsymbol{y} \\
\delta \dot{\boldsymbol{y}} \\
\delta \ddot{\boldsymbol{y}} \\
\vdots \\
d^{k-1} \delta \boldsymbol{y} / d t^{k-1}
\end{array}\right]=O_{k} \delta \boldsymbol{x}
$$

where the observability matrix takes the form

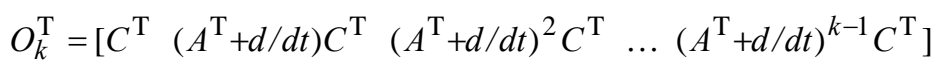

Given a known left-hand side of Eq. (5) based on the known measurements and their inferred time derivatives, the observability of the system can be analyzed by considering the uniqueness of the $\delta \boldsymbol{x}$ solution of Eq. (5). The solution is unique and the system is observable if all 13 columns of $O_{k}$ are linearly independent. If there are fewer than 13 linearly independent columns for all values of $k$, then no solution to Eq. (13) is unique, and the system is not observable.

The number of rows in $O_{k}$ is $3 k$, and the minimum $k$ for which column linear independence is possible is $k=5$. Using Eqs. (6), (2), (3), and (4a) along with the definitions of the matrices $A(t)$ and $C$, the $15 \times 13 O_{5}$ matrix takes the form:

$$
O_{5}=\left[\begin{array}{ccccc}
I & 0 & -I & 0 & 0 \\
0 & I & 0 & -I & 0 \\
G_{1} & 0 & -G_{2} & 0 & \gamma_{1}-\gamma_{2} \\
\dot{G}_{1} & G_{1} & -\dot{G}_{2} & -G_{2} & \dot{\gamma}_{1}-\dot{\gamma}_{2} \\
\ddot{G}_{1}+G_{1}^{2} & 2 \dot{G}_{1} & -\ddot{G}_{2}-G_{2}^{2} & -2 \dot{G}_{2} & \ddot{\gamma}_{1}+G_{1} \gamma_{1}-\ddot{\gamma}_{2}-G_{2} \gamma_{2}
\end{array}\right]
$$

where

$$
\begin{aligned}
& \dot{G}_{i}=\frac{3 \mu}{\left\|\boldsymbol{r}_{i}\right\|^{4}}\left[\boldsymbol{v}_{i} \hat{\boldsymbol{r}}_{i}^{\mathrm{T}}+\hat{\boldsymbol{r}}_{i} \boldsymbol{v}_{i}^{\mathrm{T}}-\left(\hat{\boldsymbol{r}}_{i}^{\mathrm{T}} \boldsymbol{v}_{i}\right)\left(5 \hat{\boldsymbol{r}}_{i} \hat{\boldsymbol{r}}_{i}^{\mathrm{T}}-I\right)\right] \quad \text { for } i=1,2 \\
& \ddot{G}_{i}=\frac{3 \mu}{\left\|\boldsymbol{r}_{i}\right\|^{5}}\left[5\left(\hat{\boldsymbol{r}}_{i}^{\mathrm{T}} \boldsymbol{v}_{i}\right)^{2}\left(7 \hat{\boldsymbol{r}}_{i} \hat{\boldsymbol{r}}_{i}^{\mathrm{T}}-I\right)-10\left(\hat{\boldsymbol{r}}_{i}^{\mathrm{T}} \boldsymbol{v}_{i}\right)\left(\boldsymbol{v}_{i} \hat{\boldsymbol{r}}_{i}^{\mathrm{T}}+\hat{\boldsymbol{r}}_{i} \boldsymbol{v}_{i}^{\mathrm{T}}\right)+2 \boldsymbol{v}_{i} \boldsymbol{v}_{i}^{\mathrm{T}}-\left(\boldsymbol{v}_{i}^{\mathrm{T}} \boldsymbol{v}_{i}\right)\left(5 \hat{\boldsymbol{r}}_{i} \hat{\boldsymbol{r}}_{i}^{\mathrm{T}}-I\right)+\left(\boldsymbol{r}_{i}^{\mathrm{T}} \boldsymbol{r}_{i}\right) G_{i}\right] \\
& \dot{\gamma}_{i}=\frac{1}{\mu} G_{i} \boldsymbol{v}_{i} \quad \text { and } \quad \ddot{\gamma}_{i}=\frac{1}{\mu} \dot{G}_{i} \boldsymbol{v}_{i}+G_{i} \boldsymbol{\gamma}_{i} \quad \text { for } i=1,2
\end{aligned}
$$




\section{B. Observability Analysis of Possible $O_{k}$ Null-Space}

In keeping with the analysis of the appendix of ${ }^{8}$, the condition for observability can be expressed as follows: The system is observable if and only if the system of equations:

$$
O_{k} \delta \mathbf{x}=0
$$

only has the trivial solution $\delta \mathbf{x}=0$ for all $k \geq 5$. Thus, the question of observability reduces to the question of whether any of the matrices $O_{5}, O_{6}, O_{7}, \ldots$ has only a trivial null space. Given that the first $3 k$ rows of $O_{k+1}$ are equal to $O_{k}$, one way to confirm observability is to assume that all of the $O_{k}$ matrices have a non-trivial null space and to show that this assumption leads to a contradiction. Equivalently, one can assume that the columns of each $O_{k}$ matrix are linearly dependent and then show that this leads to a contradiction. This is the method used in ${ }^{8}$ to determine which cases are observable and which cases are not observable.

All of the unobservable cases of ${ }^{8}$ are also unobservable in the present situation. This is true because the first 12 columns of each new $O_{k}$ matrix equal the corresponding $O_{k}$ matrix of ${ }^{8}$. The unobservable cases found in ${ }^{8}$ correspond to a non-trivial intersection of all the non-trivial null spaces of all of the original analysis' $O_{k}$ matrices. This same subspace will be a non-trivial intersection of the null spaces in the present context because the required non-trivial $\delta \boldsymbol{x}$ null-space vectors can be constructed from those of ${ }^{8}$ simply by appending the trivial $13^{\text {th }}$ element $\delta \mu$ $=0$. Thus, the following two unobservable cases from ${ }^{8}$ remain unobservable when the $\delta \mu$ gravity correction needs to be estimated along with the two orbits: Both unobservable cases are for elliptical orbits with the same semimajor axis, the same eccentricity, and the same mean anomaly at the same epoch. These conditions cause them to have identical altitude and speed time histories. If the two orbits are also co-planar, then they are unobservable. Alternatively, if they are non-coplanar, then they are still unobservable if they are phased to cross the intersection of the orbital planes at the same time (which leads to the impractical implication that they collide twice per orbit).

The question of present interest concerns whether additional cases are unobservable. This question can be addressed after reducing the dimensions of the matrices that must be considered. The first 6 rows of Eq. (9) for any $k \geq 2$ imply that $\delta \boldsymbol{r}_{1}=\delta \boldsymbol{r}_{2}$ and $\delta \boldsymbol{v}_{1}=\delta \boldsymbol{v}_{2}$ in any null space of $O_{k}$. Thus, the unobservability condition in Eq. (9) can be reduced to

$$
\left[\begin{array}{ll}
0 & I
\end{array}\right] O_{k}\left[\begin{array}{ccc}
I & 0 & 0 \\
0 & I & 0 \\
I & 0 & 0 \\
0 & I & 0 \\
0 & 0 & 1
\end{array}\right]\left[\begin{array}{l}
\delta \boldsymbol{r} \\
\delta \boldsymbol{v} \\
\delta \mu
\end{array}\right]=0=\widetilde{O}_{k} \delta \tilde{\mathbf{x}} \text { where } \widetilde{O}_{k}=\left[\begin{array}{ll}
0 & I
\end{array}\right] O_{k}\left[\begin{array}{ccc}
I & 0 & 0 \\
0 & I & 0 \\
I & 0 & 0 \\
0 & I & 0 \\
0 & 0 & 1
\end{array}\right] \text { and } \delta \tilde{\mathbf{x}}=\left[\begin{array}{c}
\delta \boldsymbol{r} \\
\delta \boldsymbol{v} \\
\delta \mu
\end{array}\right]
$$

In Eq. (10), $\delta \boldsymbol{r}=\delta \boldsymbol{r}_{1}=\delta \boldsymbol{r}_{2}$ is the common position perturbation of the 2 satellites, $\delta \boldsymbol{v}=\delta \boldsymbol{v}_{1}=\delta \boldsymbol{v}_{2}$ is their common velocity perturbation, and $\widetilde{O}_{k}$ is a $3(k-2)$-by-7 reduced observability matrix. Note that the matrix [0 I] on the extreme left-hand side of Eq. (10) is a $3(k-2)$ by $3 k$ matrix that acts to delete the first 6 rows from $O_{k}$ in order to produce $\widetilde{O}_{k}$. As an example, the reduced observability matrix for $k=5$ is the 9-by-7 matrix

$$
\widetilde{O}_{5}=\left[\begin{array}{ccc}
G_{1}-G_{2} & 0 & \gamma_{1}-\gamma_{2} \\
\dot{G}_{1}-\dot{G}_{2} & G_{1}-G_{2} & \dot{\gamma}_{1}-\dot{\gamma}_{2} \\
\ddot{G}_{1}+G_{1}^{2}-\ddot{G}_{2}-G_{2}^{2} & 2\left(\dot{G}_{1}-\dot{G}_{2}\right) & \ddot{\gamma}_{1}+G_{1} \gamma_{1}-\ddot{\gamma}_{2}-G_{2} \gamma_{2}
\end{array}\right]
$$

If the columns of $\widetilde{O}_{k}$ are linearly independent for any value of $k$, then the system is observable for the given pair of orbits. If $\widetilde{O}_{k}$ has a non-trivial null space for all $k$, then the system is unobservable. Note that the null-space of $\widetilde{O}_{k}$ is necessarily contained in the null space of $\widetilde{O}_{j}$ for all $j<k$ because $\widetilde{O}_{k}$ is created from $\widetilde{O}_{j}$ by appending $3(k-j)$ additional rows.

\section{Numerical Observability Analysis of $\widetilde{\boldsymbol{O}}_{5}$}

The present analysis departs from that of ${ }^{8}$ because it has not developed a comprehensive analytical result that deals with all $\widetilde{O}_{k}$ for $k=3,4,5, \ldots$ in order to determine all observable and unobservable cases. Instead, a numerical study of the system's observability has been performed by considering the linear independence of the columns of $\widetilde{O}_{5}$. This is a reasonable initial approach because $\widetilde{O}_{5}$ has enough rows to allow the possibility of its columns being linearly independent and because the analysis of ${ }^{8}$ found that the linear dependence or independence 
of the columns of $\widetilde{O}_{5}$ carried through to $\widetilde{O}_{k}$ for all $k>5$.

The choice of the scaling of the columns and rows of $\widetilde{O}_{k}$ becomes important in a numerical observability analysis. The finite precision of computer calculations can cause false determinations of linear dependence or linear independence if a poor scaling is chosen. A reasonable numerical scaling of $\widetilde{O}_{k}$ can be achieved if all lengths are expressed in units of radii of the central body and if all times are expressed in units of Hergs, where one Herg equals $\left(a_{b}^{3} / \mu\right)^{0.5}$ seconds if the radius of the central body $a_{b}$ is expressed in meters and if its gravitational constant $\mu$ is expressed in $\mathrm{m}^{3} / \mathrm{s}^{2}$. In this alternate system of units, $\mu=1 \mathrm{radius}^{3} / \mathrm{Herg}^{2}$, and $2 \pi$ Hergs is the period of an orbit whose semi-major axis equals the attracting body's radius.

The results of this numerical analysis indicate that the system is observable in the general case because $\widetilde{O}_{5}$ has full column rank. There is, however, a new unobservable case: Like the two unobservable cases of ${ }^{8}$, both spacecraft orbits have identical altitude and speed time histories, i.e., $\left\|\boldsymbol{r}_{1}(t)\right\|=\left\|\boldsymbol{r}_{2}(t)\right\|$ and $\left\|\boldsymbol{v}_{1}(t)\right\|=\left\|\boldsymbol{v}_{2}(t)\right\|$ for all $t$. This occurs only if they have the same semi-major axis, the same eccentricity, and the same mean anomaly at epoch. Different from the situation of ${ }^{8}$, however, this new unobservable case does not require that the orbital planes be the same, or that the orbits be phased so that the two spacecraft cross the intersection of non-coplanar orbits at the same time. The non-trivial unobservable displacement $\delta \boldsymbol{r}=\delta \boldsymbol{r}_{1}=\delta \boldsymbol{r}_{2}$ is perpendicular to the nominal relative position vector $\left(\boldsymbol{r}_{1}-\boldsymbol{r}_{2}\right)$ in this new unobservable case, but the non-trivial unobservable velocity $\delta \boldsymbol{v}=\delta \boldsymbol{v}_{1}=\boldsymbol{\delta}_{2}$ can have a component along this direction. This counter-intuitive result has yet to be explained.

The general observable case includes the situation in which both satellite orbits have the same semi-major axis and eccentricity. In this case, observability holds true if the two orbits' mean anomalies at epoch differ. This difference causes their altitude and speed time histories to differ by a time lag. This case remains observable even when the 2 orbits are coplanar.

\section{Problem Model with High-Order Gravity Terms and Realistic Measurements}

The goal of this section is to develop the state, dynamics, and measurement models of a realistic estimation problem for the two spacecraft orbits and for corrections to a spherical harmonic gravity model. This problem model will be used to design an EKF that estimates these quantities, to derive a numerical analysis of their observability, and to develop a truth-model simulation that can be used to study the Kalman filter's performance.

\section{A. Gravity Model}

The general non-spherical gravitational model used in this study takes the form

$$
\boldsymbol{g}(\boldsymbol{r}, t)=A_{i b}(t) \boldsymbol{g}_{b}\left[A_{i b}^{\mathrm{T}}(t) \boldsymbol{r} ; \mu, C_{20}, C_{21}, S_{21}, C_{22}, S_{22}, C_{30}, \ldots, S_{N N}\right]+\sum_{j=1}^{J} \Delta \boldsymbol{g}_{o j}(\boldsymbol{r}, t)
$$

where $\boldsymbol{g}(\boldsymbol{r}, t)$ is the gravitational acceleration at position $\boldsymbol{r}$ and time $t$. Both $\boldsymbol{g}$ and $\boldsymbol{r}$ are measured with respect to coordinates whose origin is fixed at the primary attracting body's center of mass and whose axes are inertially aligned.

The gravitational acceleration in Eq. (12) consists of a term due to the gravity of the primary attracting body, the $A_{i b}(t) \boldsymbol{g}_{b}$ term, and terms due to the gravity of $J$ nearby attracting bodies, the $\Delta \boldsymbol{g}_{o j}(\boldsymbol{r}, t)$ terms for $j=1, \ldots, J$. The former term is the product of the direction cosines rotation matrix $A_{i b}(t)$, which transforms from attracting-bodyfixed coordinates to inertial coordinates, and the gravity as computed in the body-fixed coordinate system, $\boldsymbol{g}_{b}$. The $A_{i b}(t)$ body attitude matrix is assumed to be a known function of time. Each of the $\Delta \boldsymbol{g}_{o j}(\boldsymbol{r}, t)$ terms is the difference between the nearby body's gravitational acceleration at $\boldsymbol{r}$ and its acceleration at the center of mass of the primary attracting body, hence the $\Delta$ prefix for each of these terms. These terms depend on time because of the motions of the secondary bodies relative to the primary body. $J=2$ for Lunar orbit/gravity determination problems, with the Earth and the Sun being the two bodies of interest. Their effects are modeled using simple $1 / r^{2}$ gravitation laws.

The gravitational acceleration of the non-spherical mass distribution of the primary attracting body depends on the satellite's location in body-fixed coordinates, $\boldsymbol{r}_{b}=A_{i b}^{\mathrm{T}}(t) \boldsymbol{r}$, and on the $N^{2}+2 N-2$ gravity model parameters $\mu, C_{20}$, $C_{21}, S_{21}, \ldots, S_{N N}$. The parameters $C_{20}$ through $S_{N N}$ are the coefficients in an $N$-degree, $N$-order spherical harmonic approximation of the body's gravitational potential energy per unit mass ${ }^{10}$ : 


$$
U_{b}\left(\boldsymbol{r}_{b}\right)=\frac{\mu}{\left\|\boldsymbol{r}_{b}\right\|}\left\{-1+\sum_{n=2}^{N}\left(\frac{a_{b}}{\left\|\boldsymbol{r}_{b}\right\|}\right)^{n} \sum_{m=0}^{n} P_{n m}\left[\sin \theta\left(\boldsymbol{r}_{b}\right)\right]\left\{C_{n m} \cos \left[m \lambda\left(\boldsymbol{r}_{b}\right)\right]+S_{n m} \sin \left[m \lambda\left(\boldsymbol{r}_{b}\right)\right]\right\}\right\}
$$

where the functions $P_{n m}[]$ are Legendre and associated Legendre functions, $\theta\left(\boldsymbol{r}_{b}\right)$ is the body-relative latitude at the position $\boldsymbol{r}_{b}$, and $\lambda\left(\boldsymbol{r}_{b}\right)$ is the body-relative longitude at $\boldsymbol{r}_{b}$.

The gravitational acceleration of the central attracting body is computed from this potential energy function by taking its gradient:

$$
\boldsymbol{g}_{b}\left(\boldsymbol{r}_{b} ; \mu, C_{20}, C_{21}, S_{21}, C_{22}, S_{22}, C_{30}, \ldots, S_{N N}\right)=-\nabla U_{b}=-\left[\frac{\partial U_{b}}{\partial \boldsymbol{r}_{b}}\right]^{\mathrm{T}}
$$

The actual computation of this gradient is carried out using the non-singular recursion II of Table 2 of ${ }^{10}$.

The EKF and the observability analysis also require the derivatives of $\boldsymbol{g}_{b}$ with respect to $\boldsymbol{r}_{b}, \mu, C_{20}, C_{21}, S_{21}, \ldots$, and $S_{N N}$. These derivatives are needed in order to estimate both the orbits and corrections to the gravity model parameters. These derivatives are computed by using the chain rule in term-by-term differentiation of the recursion that is used to compute $\boldsymbol{g}_{b}$.

\section{B. Estimated State Vector}

This paper's Kalman filter estimates the following state vector:

$$
\boldsymbol{x}=\left[\boldsymbol{r}_{1}^{\mathrm{T}}, \boldsymbol{v}_{1}^{\mathrm{T}}, \delta \boldsymbol{a}_{1}^{\mathrm{T}}, \beta_{1}, \boldsymbol{r}_{2}^{\mathrm{T}}, \boldsymbol{v}_{2}^{\mathrm{T}}, \delta \boldsymbol{a}_{2}^{\mathrm{T}}, \beta_{2}, \Delta \rho_{\phi}, \Delta \rho_{p}, \mu, C_{20}, C_{21}, S_{21}, C_{22}, S_{22}, C_{30}, \ldots, S_{N N}\right]^{T}
$$

where $\delta \boldsymbol{a}_{1}$ and $\delta \boldsymbol{a}_{2}$ are 3-dimensional disturbance acceleration vectors that act on satellites 1 and 2, $\beta_{1}$ and $\beta_{2}$ are solar radiation pressure coefficients in units of area divided by mass for satellites 1 and $2, \Delta \rho_{\phi}$ is the bias in a measurement of the accumulated delta range between the satellites that is based on the carrier phase of a radio crosslink, and $\Delta \rho_{p}$ is the bias in a measurement of the absolute range between the satellites that is based on a transmitted pseudo-random number (PRN) code, much like GPS pseudorange measurements. The dimension of this state vector $N^{2}+2 N+20$.

It would be possible to eliminate the need to estimate the solar radiation pressure coefficients $\beta_{1}$ and $\beta_{2}$ if the satellites carried accurate accelerometers and attitude sensors. These sensors could be used to directly measure the non-gravitational forces, which could then be used in the filter's dynamic model. Note, however, that the random acceleration errors, $\delta \boldsymbol{a}_{1}$ and $\delta \boldsymbol{a}_{2}$, might still be needed in order to account for the effects of unmodeled higher-order terms in the actual gravity field. If the orbits were near the Earth and if accelerometer data were not available, then one would need to estimate atmospheric drag parameters that were similar to $\beta_{1}$ and $\beta_{2}$. Drag parameters have been omitted because the target application is Lunar orbits, where drag is negligible.

\section{Dynamics Model}

This sub-section presents the various equations that the Kalman filter uses to model the time evolution of its state vector. The time evolution of the position and velocity of each satellite is modeled using its point-mass translational equations of kinematics and dynamics:

$$
\left[\begin{array}{c}
\dot{\boldsymbol{r}}_{i} \\
\dot{\boldsymbol{v}}_{i}
\end{array}\right]=\left[\begin{array}{c}
\boldsymbol{v}_{i} \\
\boldsymbol{g}\left(\boldsymbol{r}_{i}, t\right)+\delta \boldsymbol{a}_{i}+\beta_{i} p_{S}\left(\boldsymbol{r}_{i}, \boldsymbol{r}_{S}\right)\left(\boldsymbol{r}_{i}-\boldsymbol{r}_{S}\right) /\left\|\boldsymbol{r}_{i}-\boldsymbol{r}_{S}\right\|
\end{array}\right] \text { for } i=1,2
$$

where $\boldsymbol{r}_{s}$ is the position of the Sun and $p_{s}\left(\boldsymbol{r}_{i}, \boldsymbol{r}_{s}\right)$ is the solar radiation pressure, which depends on distance from the Sun and on whether or not the spacecraft is in the shadow of the primary attracting body.

The acceleration disturbances are modeled by a discrete-time first-order Gauss/Markov process:

$$
\delta \boldsymbol{a}_{i}\left(t_{k+1}\right)=e^{-\Delta t_{k} / \tau_{\text {aik }}} \delta \boldsymbol{a}_{i}\left(t_{k}\right)+\sigma_{\text {aik }} \sqrt{1-e^{-2 \Delta t_{k} / \tau_{a i k}}} \boldsymbol{v}_{\text {aik }} \text { for } i=1,2
$$

where $t_{k}$ and $t_{k+1}$ are sample times, $\Delta t_{k}=t_{k+1}-t_{k}$, and $\boldsymbol{v}_{a i k}$ is a 3-dimensional discrete-time Gaussian white-noise sequence with a mean of zero and a covariance equal to the identity matrix. The Markov process' first-order time constant $\tau_{\text {aik }}$ depends on the satellite index $i$ and the sample index $k$ because it is scaled to vary in proportion to

$\left\|r_{i}\left(t_{k}\right)\right\|^{1.5}$ in order to model the effect of altitude on the rate of change of the effects of unmodeled higher-order 
terms in the actual gravity field. Similarly, the Markov process' nominal standard deviation $\sigma_{a i k}$ depends on $i$ and $k$ because it is scaled to vary in proportion to $\left\|\boldsymbol{r}_{i}\left(t_{k}\right)\right\|^{-3}$ in order to model the effect of altitude on the magnitudes of unmodeled higher-order terms in the actual gravity field. This is a conservative model of the acceleration error magnitude's fall-off with altitude that presumes higher magnitudes of the unmodeled gravity terms at high altitudes than is likely warranted.

The dynamics of the solar radiation pressure parameters are modeled by discrete-time random-walk processes:

$$
\beta_{i}\left(t_{k+1}\right)=\beta_{i}\left(t_{k}\right)+\sigma_{\beta} \sqrt{\Delta t_{k}} v_{\beta i k} \text { for } i=1,2
$$

where $v_{\beta i k}$ is a scalar discrete-time Gaussian white-noise sequence with a mean of zero and a covariance of 1 , and $\sigma_{\beta}$ is the square-root of the intensity of the white noise process that drives the random walk.

The remaining elements of the state vector are modeled as being constants. Their discrete-time dynamic models take the forms

$$
\begin{aligned}
& \Delta \rho_{\phi}\left(t_{k+1}\right)=\Delta \rho_{\phi}\left(t_{k}\right) \\
& \Delta \rho_{p}\left(t_{k+1}\right)=\Delta \rho_{p}\left(t_{k}\right) \\
& \mu\left(t_{k+1}\right)=\mu\left(t_{k}\right) \\
& C_{20}\left(t_{k+1}\right)=C_{20}\left(t_{k}\right) \\
& \vdots \\
& S_{N N}\left(t_{k+1}\right)=S_{N N}\left(t_{k}\right)
\end{aligned}
$$

The discrete-time dynamic models for the time evolution of the satellites' positions and velocities are derived via numerical integration of the system of coupled nonlinear differential equations in Eq. (16). The 1-sample integration from time $t_{k}$ to time $t_{k+1}$ is performed using constant values for the disturbance acceleration and for the solar radiation pressure coefficient. These values are

$$
\begin{aligned}
& \delta \boldsymbol{a}_{i}=0.5\left[\delta \boldsymbol{a}_{i}\left(t_{k+1}\right)+\delta \boldsymbol{a}_{i}\left(t_{k}\right)\right] \quad \text { for } i=1,2 \\
& \beta_{i}=0.5\left[\beta_{i}\left(t_{k+1}\right)+\beta_{i}\left(t_{k}\right)\right] \quad \text { for } i=1,2
\end{aligned}
$$

The values $\delta \boldsymbol{a}_{i}\left(t_{k}\right)$ and $\delta \boldsymbol{a}_{i}\left(t_{k+1}\right)$ are taken from the discrete-time Markov model in Eq. (17), and the values $\beta_{i}\left(t_{k}\right)$ and $\beta_{i}\left(t_{k+1}\right)$ are taken from the discrete-time random-walk model in Eq. (18).

The dynamics models described in Eqs. (16)-(20b) can be assembled into the generic discrete-time model form

$$
\boldsymbol{x}_{k+1}=\boldsymbol{f}_{k}\left(\boldsymbol{x}_{k}, \boldsymbol{w}_{k}\right)
$$

where $\boldsymbol{x}_{k}$ is the state vector from Eq. (15) at sample time $t_{k}$ and $\boldsymbol{x}_{k+1}$ is the state at time $t_{k+1}$. The discrete-time process-noise vector $\boldsymbol{w}_{k}$ consists of 8 elements:

$$
\boldsymbol{w}_{k}=\left[\boldsymbol{v}_{a 1 k}^{\mathrm{T}}, \boldsymbol{v}_{\beta 1 k}, \boldsymbol{v}_{a 2 k}^{\mathrm{T}}, \boldsymbol{v}_{\beta 2 k}\right]^{T}
$$

It has a mean of zero and a covariance equal to the identity matrix.

The EKF and the observability analysis of Section IV both require computation of the dynamics model's Jacobian matrices

$$
\Phi_{k}=\left.\frac{\partial \boldsymbol{f}_{k}}{\partial \boldsymbol{x}_{k}}\right|_{\left(\boldsymbol{x}_{k}, \boldsymbol{w}_{k}\right)} \text { and } \quad \Gamma_{k}=\left.\frac{\partial \boldsymbol{f}_{k}}{\partial \boldsymbol{w}_{k}}\right|_{\left(\boldsymbol{x}_{k}, \boldsymbol{w}_{k}\right)}
$$

Computation of the elements of these matrices that are associated with Eqs. (17)-(19e) is simple. Computation of the elements associated with the $\boldsymbol{r}_{i}$ and $\boldsymbol{v}_{i}$ propagations, although straightforward, is complicated. These computations involve integration of a state transition matrix differential equation to determine the corresponding components of $\Phi_{k}$ and integration of a disturbance effectiveness matrix differential equation to compute the corresponding components of $\Gamma_{k}$. The matrix differential equation for the $\Phi_{k}$ components can be derived by differentiating the system of differential equations in Eq. (16) with respect to the initial condition $\boldsymbol{x}_{k}$, and the matrix differential equation for the $\Gamma_{k}$ components comes from differentiating the system in Eq. (16) with respect to the constant disturbance parameters $\delta \boldsymbol{a}_{i}$ or $\beta_{i}$. The computation of $\Gamma_{k}$ includes application of the chain rule in order to 
account for the effects of elements of $\boldsymbol{w}_{k}$ on $\delta \boldsymbol{a}_{i}$ and $\beta_{i}$ as implied by Eqs. (17), (18), (20a), and (20b). Note that the altitude dependence of the quantities $\tau_{a i k}$ and $\sigma_{a i k}$ in Eq. (17) has not been considered when computing $\Phi_{k}$ and $\Gamma_{k}$ because its effect on the derivatives is presumed to be negligible.

The matrices $\Phi_{k}$ and $\Gamma_{k}$ are large and have a structured sparsity when $N$, the maximum order and degree of the estimated corrections to the gravity model, is large. It is usually worthwhile in terms of computational time savings to tailor any filter calculation to exploit these matrices' structures.

\section{Measurement Model}

This section develops a model for a realistic set measurements of the relative position vector $\Delta \boldsymbol{r}_{12}$. The likely instruments that would be used to measure $\Delta \boldsymbol{r}_{12}$ would be a radio cross-link to measure the magnitude $\left\|\Delta \boldsymbol{r}_{12}\right\|$ and an optical system to measure the bearing $\Delta \hat{r}_{12}=\Delta \boldsymbol{r}_{12} /\left\|\Delta \boldsymbol{r}_{12}\right\|$.

This study presumes that the radio cross-link would measure $\left\|\Delta \boldsymbol{r}_{12}\right\|$ based on the principle of dual one-way ranging ${ }^{1}$. In this method, each satellite would have a GPS-like transmitter and a GPS-like receiver that would run off of a common oscillator. Each satellite's range measurement would include the effects of receiver/transmitter clock errors. If a stable oscillator were used on each satellite and if the range measurements from the two satellites were averaged, then the effects of the receiver/transmitter clock errors would drop out.

The measurement concept envisions using two types of range measurements that are derivable from the radio cross link. One type measures the relative change in range based on precise carrier phase measurements, and the other type measures absolute range using the time of flight of a PRN code, as in GPS pseudorange measurements. The models for these two measurement types are

$$
\begin{aligned}
& y_{1 k}=\left\|\boldsymbol{r}_{1}\left(t_{k}\right)-\boldsymbol{r}_{2}\left(t_{k}\right)\right\|+\Delta \rho_{\phi}+v_{\phi k} \\
& y_{2 k}=\left\|\boldsymbol{r}_{1}\left(t_{k}\right)-\boldsymbol{r}_{2}\left(t_{k}\right)\right\|+\Delta \rho_{p}+v_{p k}
\end{aligned}
$$

where $\Delta \rho_{\phi}$ and $\Delta \rho_{p}$ constitute measurement biases and $v_{\phi k}$ and $v_{p k}$ are uncorrelated, zero-mean, discrete-time Gaussian white noise measurement errors with the respective standard deviations $\sigma_{\phi}$ and $\sigma_{p}$. Although these two models look identical, there are important differences. The carrier-phase measurement in Eq. (24a) normally will be very precise, with $\sigma_{\phi} \cong 0.005 \mathrm{~m}$ or even less. The absolute range measurement in Eq. (24b) will be much less precise; $\sigma_{p} \cong 0.5 \mathrm{~m}$. The advantage of the latter measurement is that there will be a priori knowledge that the absolute range bias has an expected value of $E\left\{\Delta \rho_{p}\right\}=0$ with a standard deviation on the order of the residual calibration error, which might be about $0.5 \mathrm{~m}$. The $\Delta \rho_{\phi}$ bias of the carrier phase measurement, on the other hand, will have no a priori mean or standard deviation. Thus, it will have to be estimated from scratch. The first measurement has been included because it is known to be useful on the GRACE mission for recovering high-order gravity effects. The second measurement has been included because it makes the system much more likely to be observable; the observability calculations of ${ }^{8,9}$ and of Section II presume the availability of an absolute range measurement.

This study assumes that the relative direction vector $\Delta \hat{r}_{12}$ would be measured by an optical system. Suppose that Satellite 1 carried a light beacon, perhaps a bright light-emitting diode, and that Satellite 2 carried a star camera. The intensity of the light beacon could be sized to enable the star camera to detect it at typical inter-satellite distances, which may range from 10 to $50 \mathrm{~km}$. The beacon could flash on and off, both to save power and to help the star camera distinguish it from stars. The star camera's data processing software could be modified to enable it to identify the beacon and locate the beacon relative to the stars. Measurement of the location of the beacon relative to known stars in the camera's field of view (FOV) would constitute measurement of $\Delta \hat{r}_{12}$.

The relative bearing measurement model takes the form

$$
\left[\begin{array}{l}
y_{3 k} \\
y_{4 k} \\
y_{5 k}
\end{array}\right]=\Delta \hat{\boldsymbol{r}}_{12}+\boldsymbol{v}_{\Delta r k}=\frac{\boldsymbol{r}_{1}\left(t_{k}\right)-\boldsymbol{r}_{2}\left(t_{k}\right)}{\left\|\boldsymbol{r}_{1}\left(t_{k}\right)-\boldsymbol{r}_{2}\left(t_{k}\right)\right\|}+\boldsymbol{v}_{\Delta r k}
$$

where $\boldsymbol{v}_{\Delta r k}$ is the 3-dimensional uncorrelated, zero-mean, discrete-time Gaussian white noise bearing measurement error vector with covariance equal to $\sigma_{\Delta r} I$. Technically, $\boldsymbol{v}_{\Delta r k}$ should have zero variance in the direction $\Delta \hat{r}_{12}$ because the length of this vector is known exactly. Practically, this slight modeling error has no impact on the filter's performance, and it is tolerated because it simplifies the necessary computations.

American Institute of Aeronautics and Astronautics 
Equation (25) includes no measurement bias term. The use of a single star camera to measure both the beacon and the inertial direction of the stars tends to cancel out the effects of biases if the camera's optics and CCD sensor exhibit no significant geometric nonlinearities. If one instrument were used to measure Satellite 2's inertial attitude and if a second instrument were used to measure the bearing of the Satellite-1 beacon relative to Satellite 2's bodyfixed coordinates, then some sort of alignment bias would be present in the resultant $\Delta \hat{r}_{12}$ measurement. Therefore, the best instrumentation strategy is to avoid such biases by making the two measurements using a single instrument, as envisioned in the present study.

The bearing measurement $\Delta \hat{r}_{12}$ may not be available continuously. Satellite 2 might be required to perform slew maneuvers in order to keep Satellite 1's beacon in the star camera's FOV. Alternatively, the star camera might be mounted on a gimbaled platform so that it could be slewed without having to maneuver the whole spacecraft. In either case, it might not always be possible or practical to slew the camera FOV to keep Satellite 1 in view. Two additional problems with this measurement are glare and lack of stars in the FOV. If the Sun, the Earth, or the sunlight portion of the primary attracting body fell within the FOV, then the camera could not be used because of too much glare. Alternatively, if the dark side of the primary attracting body took up all or most of the FOV, then there would be no stars to provide an inertial point of reference. This latter situation will arise whenever $\Delta \hat{r}_{12}$ is aligned too closely with the nadir vector from Satellite 2. Therefore, this system must be able to cope with intermittent unavailability of the $\Delta \hat{r}_{12}$ measurement.

An additional measurement is considered at the end of this study as a means of enhancing the observability of the central gravity parameter $\mu$. This measurement is the range rate of one of the spacecraft relative to an Earthbased tracking station. This measurement could be made using the carrier phase part of a dual-one-way-ranging system between the spacecraft and the tracking station. It is envisioned that such a measurement would be used infrequently, perhaps only once a month with only a $100 \mathrm{sec}$ tracking period. Such data, although violating the goal of complete autonomy, might greatly enhance the system's ability to perform its orbit/gravity estimation task. If the range rate measurement is made for Satellite 2, then the model for this measurement takes the form:

$$
y_{6 k}=\Delta \hat{\boldsymbol{r}}_{2 e k}^{\mathrm{T}} \boldsymbol{v}_{2}\left(t_{k}\right)+v_{v 2 k}
$$

where $\Delta \hat{r}_{2 e k}$ is the unit direction vector from the Earth tracking station to Satellite 2 and $v_{v 2 k}$ is the zero-main, Gaussian measurement error whose standard deviation is $\sigma_{v 2}$. This simplified measurement model presumes that $\Delta \hat{\boldsymbol{r}}_{2 e k}$ is known exactly, which is not quite true due to uncertainty in $\boldsymbol{r}_{2}$, but the uncertainty is small enough to neglect. This model lumps the effect of the Earth tracking station's Moon-relative velocity into the measurement $y_{6 k}$.

In summary, the measurements in Eqs. (24a)-(26) can be lumped into a single nonlinear measurement equation of the form:

$$
\boldsymbol{y}_{k}=\boldsymbol{h}_{k}\left(\boldsymbol{x}_{k}\right)+\boldsymbol{v}_{k}
$$

where $\boldsymbol{y}_{k}$ is the measurement vector that is composed of the left-hand sides of Eqs. (24a)-(26), $\boldsymbol{h}_{k}\left(\boldsymbol{x}_{k}\right)$ is the measurement model function whose components are the leading terms on the right-hand sides of Eqs. (24a)-(26), and $\boldsymbol{v}_{k}$ is the measurement noise vector whose elements are the noise terms in Eqs. (24a)-(26). If $R_{v v k}$ is defined to be the square-root information matrix for the random measurement error vector $\boldsymbol{v}_{k}$, then $R_{v v k}^{-1}=$ $\operatorname{diag}\left(\sigma_{\phi}, \sigma_{p}, \sigma_{\Delta r}, \sigma_{\Delta r}, \sigma_{\Delta r}, \sigma_{v 2}\right)$. The Jacobian matrix of the measurement function is defined to be $H_{k}=\partial \boldsymbol{h}_{k} / \partial \boldsymbol{x}_{k}$. It is needed by the Kalman filter and by the observability analysis.

The vector function $\boldsymbol{h}_{k}\left(\boldsymbol{x}_{k}\right)$ depends on $k$. As defined above, its nominal dimension is 6, but the actual dimension of $\boldsymbol{h}_{k}\left(\boldsymbol{x}_{k}\right)$ can vary with time. Its dimension will usually be 5 because the velocity measurement in Eq. (26) will be used only rarely, if ever. The number of measurements will drop down further to two whenever Satellite 2's star camera FOV fails to track the Satellite-1 beacon, whenever there is glare in the camera FOV, and whenever the FOV sees too much of the primary attracting body. This possibility of change in the number of measurements is the reason why the measurement error vector's square-root information matrix $R_{v v k}$ can vary with $k$; its dimension and element definitions must vary with those of $\boldsymbol{h}_{k}\left(\boldsymbol{x}_{k}\right)$. A second reason for variation of the function $\boldsymbol{h}_{k}\left(\boldsymbol{x}_{k}\right)$ with the 
sample index $k$ is that $\Delta \hat{r}_{2 e k}$ in Eq. (26) varies with $k$ due to the motion of the Earth relative to the primary attracting body.

\section{An EKF in SRIF form and a Linearized Observability Calculation}

The EKF that estimates the state vector of Eq. (15) and the analysis of the observability of this state both rely on linearizations of the dynamics model in Eq. (21) and the measurement model in Eq. (27). They both use standard extended SRIF techniques that are an extension of the linear SRIF developed in ${ }^{11}$. This section briefly reviews the necessary computations.

\section{A. SRIF Form of the EKF}

The SRIF form of the Kalman filter uses an information equation format to store the state estimate and its covariance. The following form of the a posteriori state information equation is suitable for use in an extended SRIF:

$$
\hat{R}_{x x k}\left(\boldsymbol{x}_{k}-\hat{\boldsymbol{x}}_{k}\right)=-\hat{\boldsymbol{v}}_{x k}
$$

where $\hat{\boldsymbol{x}}_{k}$ is the a posteriori (i.e., filtered) state estimate at sample time $t_{k}, \hat{R}_{x x k}$ is its square, upper-triangular square-root information matrix, and $\hat{\boldsymbol{v}}_{x k}$ is a discrete-time, zero-mean, Gaussian white-noise sequence with covariance equal to the identity matrix. The estimation error covariance for $\hat{\boldsymbol{x}}_{k}$ is $\hat{P}_{x x k}=\hat{R}_{x x k}^{-1} \hat{R}_{x x k}^{-\mathrm{T}}$, where the notation ()$^{-\mathrm{T}}$ indicates the inverse transpose of the matrix (). Note how the use of the overstrike $(\wedge)$ in this section indicates quantities associated with the a posteriori estimate; in previous sections it indicated unit-normalization, as for the inter-spacecraft bearing vector $\Delta \hat{r}_{12}$.

The dynamic propagation from $t_{k}$ to $t_{k+1}$ uses a linearization of Eq. (21) about the values $\boldsymbol{x}_{k}=\hat{\boldsymbol{x}}_{k}$ and $\boldsymbol{w}_{k}=0$. It performs the following computations:

$$
\begin{aligned}
& \overline{\boldsymbol{x}}_{k+1}=\boldsymbol{f}_{k}\left(\hat{\boldsymbol{x}}_{k}, 0\right) \\
& Q_{p r k}\left[\begin{array}{cc}
\bar{R}_{w w k} & \bar{R}_{w x k} \\
0 & \bar{R}_{x x k+1}
\end{array}\right]=\left[\begin{array}{cc}
I & 0 \\
-\hat{R}_{x x k} \Phi_{k}^{-1} \Gamma_{k} & \hat{R}_{x x k} \Phi_{k}^{-1}
\end{array}\right]
\end{aligned}
$$

in order to compute the a priori (i.e., predicted) state estimate $\overline{\boldsymbol{x}}_{k+1}$ and its estimation error square-root information matrix $\bar{R}_{x x k+1}$ that are valid at sample time $t_{k+1}$. The matrices on the left-hand side of Eq. (29b) are calculated using orthonormal/upper-triangular (QR) factorization of the matrix on the right-hand side of the equation ${ }^{12}$, which implies that $Q_{p r k}$ is a square, orthonormal matrix and that $\bar{R}_{w w k}$ and $\bar{R}_{x x k+1}$ are square, upper-triangular matrices. The matrix in the upper left-hand entry of the block matrix on the right-hand side of Eq. (29b) is an identity matrix because that is the correct a priori square-root information matrix for the process noise vector $\boldsymbol{w}_{k}$ as defined in the present problem. These operations result in an a priori information equation at sample time $t_{k+1}$ of the form

$$
\bar{R}_{x x k+1}\left(\boldsymbol{x}_{k+1}-\overline{\boldsymbol{x}}_{k+1}\right)=-\overline{\boldsymbol{v}}_{x k+1}
$$

where $\overline{\boldsymbol{v}}_{x k+1}$ is the information equation's zero-mean, identity-covariance noise term.

The measurement update at time $t_{k+1}$ is carried out using the following calculations:

$$
\begin{aligned}
& Q_{u p k+1}\left[\begin{array}{c}
\hat{R}_{x x k+1} \\
0
\end{array}\right]=\left[\begin{array}{c}
\bar{R}_{x x k+1} \\
R_{v v k+1} H_{k+1}
\end{array}\right] \\
& \hat{\boldsymbol{x}}_{k+1}=\overline{\boldsymbol{x}}_{k+1}+\left[\begin{array}{ll}
\hat{R}_{x x k+1}^{-1} & 0
\end{array}\right] Q_{u p k+1}^{\mathrm{T}}\left[R_{v v k+1}\left\{\boldsymbol{y}_{k+1}-\boldsymbol{h}_{k+1}\left(\overline{\boldsymbol{x}}_{k+1}\right)\right\}\right]
\end{aligned}
$$

Again, the matrices on the left-hand side of Eq. (31a) are computed via QR factorization of the matrix on the righthand side so that $Q_{u p k+1}$ is orthonormal and $\hat{R}_{x x k+1}$ is upper-triangular. The outputs of Eqs. (31a) and (31b) are $\hat{\boldsymbol{x}}_{k+1}$, the a posteriori state estimate at time $t_{k+1}$, and its estimation error square-root information matrix $\hat{R}_{x x k+1}$. These outputs complete the filter cycle for one sample interval and prepare it for recursive operation over the next sample interval. 


\section{B. SRIF Form of the Linearized Observability Calculations}

The linearized observability calculations use SRIF techniques to compute the square-root of a re-normalized observability Gramian. This Gramian square root is equivalent to the square-root information matrix for the error in the smoothed estimate of $\boldsymbol{x}_{0}$ under the assumption of zero process noise and zero a priori information about $\boldsymbol{x}_{0}$. If

this square-root information matrix is called $\widetilde{R}_{x x k}$ when based on the measurements that extend from sample time $t_{0}$ to sample time $t_{k}$, then its computation proceeds recursively as follows:

$$
\begin{aligned}
& \widetilde{\Phi}_{-1}=I \quad \text { and } \quad \widetilde{R}_{x x 0}=\left[\begin{array}{c}
0 \\
R_{v v 0} H_{0}
\end{array}\right] \\
& \widetilde{\Phi}_{k}=\Phi_{k} \widetilde{\Phi}_{k-1} \text { and } \widetilde{Q}_{k+1}\left[\begin{array}{c}
\widetilde{R}_{x x k+1} \\
0
\end{array}\right]=\left[\begin{array}{cc}
\widetilde{R}_{x x k} & \\
R_{v v k+1} H_{k+1} & \widetilde{\Phi}_{k}
\end{array}\right]
\end{aligned}
$$

Equation (32a) initializes the square matrices $\widetilde{\Phi}_{k-1}$ and $\widetilde{R}_{x x k}$ at sample $k=0$, and Eq. (32b) defines how $\widetilde{\Phi}_{k}$ and $\widetilde{R}_{x x k+1}$ are computed recursively from $\widetilde{\Phi}_{k-1}$ and $\widetilde{R}_{x x k}$. This recursion uses the linearized dynamic propagation model for the time interval from $t_{k}$ to $t_{k+1}$ and the linearized measurement model at time $t_{k+1}$. $\widetilde{\Phi}_{k}$ is the state transition matrix from time $t_{0}$ to time $t_{k+1}$. The $\widetilde{R}_{x x k+1}$ computation in Eq. (32b) uses QR factorization to compute the orthonormal matrix $\widetilde{Q}_{k+1}$ and the square, upper-triangular matrix $\widetilde{R}_{x x k+1}$ from the matrix on the right-hand side of the equation. The matrices $H_{k}$ and $\Phi_{k}$ used in Eqs. (32a) and (32b) are computed using the same linearizations as are used in the EKF of the preceding sub-section.

If $K$ is the last sample of a data batch, then the square symmetric matrix $\widetilde{R}_{x x K}^{\mathrm{T}} \widetilde{R}_{x x K}$ is the observability Gramian for the system. The system is observable if and only if this matrix is full-rank. This is equivalent to saying that the system is observable if and only if the smoothed covariance matrix $\widetilde{P}_{x x K}=\widetilde{R}_{x x K}^{-1} \widetilde{R}_{x x K}^{-\mathrm{T}}$ has all finite entries. This latter matrix is used as a measure of the degree of observability because the square-roots of its diagonal elements give indications of the estimation accuracy that can be expected for the corresponding elements of the state vector. Large diagonal elements occur when the Gramian is nearly singular. They indicate poor accuracy because of poor observability.

\section{Lunar Truth-Model Simulation}

A truth-model simulation has been used to generate simulated time histories of the measurements and states for an example orbit/gravity estimation problem at the Moon. Its output data have been used to evaluate the EKF of Section IV. The EKF operates on simulated measurements and produces state estimates. These estimates are then compared to the simulation's "truth" states. The EKF's linearizations about the resulting state estimates are used in the observability analysis.

The truth model implements a simulation of the dynamics model in Eq. (21) and the measurement model in Eq. (27). This simulation includes random measurement noise, as defined in Eq. (27), along with random initial estimation errors, but its dynamic model errors are not merely realizations of a random $\boldsymbol{w}_{k}$ process noise time history. Instead, they take the form of systematic model errors that may occur in a real system. The systematic model errors come in the form of extra terms in the gravity model. These extra terms expand the order and degree of the "truth" gravity model beyond the value $N$ used in the filter's gravity model as defined in Eq. (13). The extra gravity terms associated with the spherical harmonic coefficients, $C_{(N+1) 0}, C_{(N+1) 1}, S_{(N+1) 1}, \ldots$ act as the process noise. These "disturbance" coefficients have been chosen to be on the order of the corresponding coefficients of the Earth's gravity field for lack of a better idea of the values of the Moon's actual high-order gravity terms. It is hoped that these values are representative of likely Lunar gravity perturbations.

A significant feature of the truth-model simulation is its calculation of the availability of the relative bearing measurement in Eq. (25). The simulation assumes that Satellite 2's star camera is always slewed so that the relative bearing vector $\Delta \hat{r}_{12}$ lies in the center of its FOV. It also assumes that the FOV is circular with a fixed angular radius. It returns simulated measurements of $\Delta \hat{r}_{12}$ only if 4 conditions are met: 1) the Sun is not in the FOV, 2) no part of the Earth is in the FOV, 3) no part of the sunlit portion of the Lunar surface is in the FOV, and 4) less than $2 / 3$ of the FOV is occulted by the Moon. The first three conditions are no-glare conditions, and the last condition 
ensures that enough stars will be visible to supply an inertial reference for $\Delta \hat{r}_{12}$. Simulation of the first 2 conditions is straightforward. The $3^{\text {rd }}$ condition is tested by considering 500 random direction vectors that are sampled uniformly from the FOV. If any of them points towards a sunlit part of the Moon, then the $\Delta \hat{r}_{12}$ measurement is deemed unavailable. The $4^{\text {th }}$ condition is evaluated by computing the intersection of two small circles on a sphere, one being the FOV and the other being that subtended by the Moon. This computation involves numerical integration because a closed-form solution for the needed integral is not known.

\section{Observability and Filtering Results from the Truth-Model Simulation Study}

\section{A. Lunar Orbit Scenario}

The dual-spacecraft Lunar orbit scenario that has been considered has Satellites 1 and 2 both in elliptical orbits with identical apoapsis altitudes of $250 \mathrm{~km}$ and identical periapsis altitudes of $50 \mathrm{~km}$. Their nominal orbital periods are $7363 \mathrm{sec}$, and Satellite 1's altitude time history leads that of Satellite 2 by slightly more than $2 \%$ of an orbital period (156 sec), which leads to a maximum altitude difference between the spacecraft of $13.5 \mathrm{~km}$. Satellite 1's orbit is inclined 89.20 deg with respect to the Lunar equator, which is defined to lie in the plane of the Moon's orbit about the Earth, and Satellite 2's orbit is inclined by 89.55 deg. The total angular separation between the two orbital planes is 0.42 deg because some additional separation occurs due to a difference in the longitudes of their ascending nodes.

The truth-model simulation has been run for 336 orbits or 28.6 Earth days, that is, about one month. This length of simulation has been chosen in hopes of achieving good observability by allowing the Moon to rotate one full revolution. This rotation causes its entire surface to pass near the two orbits' periapsis, which remain almost fixed in inertially oriented coordinates. It is conjectured that this complete "scanning" of the Lunar surface by the periapsis will enhance observability of the gravity parameters because periapsis presumably is the location that offers the maximum sensitivity to the gravity parameters.

The relative position sensors are assumed to sample 100 times per orbit, or once every $73.6 \mathrm{sec}$. The simulated FOV angular radius of the star/beacon camera on Satellite 2 is 15 deg. This radius and the geometries and phasing of the orbits results in a $28 \%$ drop-out rate of the $\Delta \hat{r}_{12}$ bearing measurements due either to glare or to Lunar occultation of the stellar reference points.

\section{B. Observability Results}

The observability analysis of Section IV.B has been applied to this scenario under the assumption of complete autonomy. Autonomy presumes that there are no absolute Doppler measurements from an Earth-based tracking station, as in Eq. (26).

The state is completely observable in this situation. This case falls within the set of situations that are predicted to produce observability by the analysis of Section II. Nevertheless, the complete observability of the state in Eq. (15) is somewhat surprising given that it includes biases on both of its inter-satellite range measurements; the analysis of Section II presumes that the magnitude of $\Delta \boldsymbol{r}_{12}$ is measured without any bias.

The central-force gravity parameter $\mu$ is not strongly observable in this completely autonomous scenario. Its predicted batch estimation error standard deviation is 13.5 parts in a million. An improved accuracy would result if the estimator had access to one or more Doppler shift measurements from an Earth-based tracking station, as in Eq. (26), during the one month data collection interval.

\section{EKF Performance}

The EKF has been run several times on the data from the Lunar orbit scenario of Section VI.A. Each run has attempted to estimate gravity model corrections out to degree and order $N=12$, which yields a state vector dimension of 188. The truth-model simulation included gravity terms out to degree and order 20. One run assumed perfect a priori knowledge of $\mu$, and another run started with an initial $\mu$ error of 52 parts in $10^{6}$.

All runs have been initialized as follows: They have started with zero a priori knowledge about the carrier phase range bias state $\Delta \rho_{\phi}$ and with an a priori calibration error standard deviation of $1.5 \mathrm{~m}$ for the PRN code phase range bias state $\Delta \rho_{p}$. The solar radiation pressure parameters $\beta_{1}$ and $\beta_{2}$, the range biases $\Delta \rho_{\phi}$ and $\Delta \rho_{p}$, and all of the gravity model's spherical harmonic coefficients $C_{20}$ through $S_{N N}$ have been initialized at zero in all of the EKF runs even though the truth values for these quantities are all non-zero. 
Each run has used the tuning parameter values:

$$
\begin{aligned}
& \tau_{\text {aik }}=(175 \mathrm{sec}) \times\left[\left\|\boldsymbol{r}_{i k}\right\| /\left(1.89 \times 10^{6} \mathrm{~m}\right)\right]^{1.5}, \sigma_{a i k}=\left(10^{-7} \mathrm{~m} / \mathrm{s}^{2}\right) \times\left[\left(1.89 \times 10^{6} \mathrm{~m}\right) /\left\|\boldsymbol{r}_{i k}\right\|\right]^{3}, \\
& \sigma_{\beta}=9.18 \times 10^{-5} \mathrm{~m}^{2} /\left(\operatorname{kg} \sec ^{0.5}\right), \sigma_{\phi}=0.005 \mathrm{~m}, \sigma_{p}=1.2 \mathrm{~m}, \text { and } \sigma_{\Delta r}=5 \mathrm{arc} \mathrm{sec}
\end{aligned}
$$

The $\tau_{a i k}$ and $\sigma_{a i k}$ values in Eq. (33a) have been chosen to cause the Markov acceleration error model in Eq. (17) to give a reasonably good approximation to the acceleration errors that are caused by the filter's neglect of the higherorder terms in the truth gravity model. These values have been tuned to give the closest Markov approximation of the neglected terms when operating at the lowest orbital altitudes. Above these altitudes, the Markov model is conservative in that it presumes too much disturbance acceleration. Note that the values for $\sigma_{\phi}, \sigma_{p}$, and $\sigma_{\Delta r}$ in Eq. (33b) are the same as the values used to generate the random measurement errors in the truth-model simulation.

The results for a filter run that uses perfect a priori knowledge of $\mu$ are presented in Fig. 2. These results show a fairly rapid position and velocity convergence from initial absolute position errors of $100 \mathrm{~m}$ and initial absolute velocity errors of $0.100 \mathrm{~m} / \mathrm{s}$ to position errors on the order of $10-20 \mathrm{~m}$ and velocity errors on the order of 0.01 to $0.02 \mathrm{~m} / \mathrm{s}$. After 336 orbits, these numbers improve even further to yield per-axis RMS position errors of only $3 \mathrm{~m}$ and per-axis RMS velocity errors of only $0.003 \mathrm{~m} / \mathrm{s}$.

The estimation results for the gravity corrections are similarly encouraging. The a priori per-axis RMS gravity model error along the orbits is $4.2 \times 10^{-5} \mathrm{~m} / \mathrm{s}^{2}$. After processing the month's worth of $\Delta \boldsymbol{r}_{12}$ data, this error level is reduced to $2.7 \times 10^{-7} \mathrm{~m} / \mathrm{s}^{2}$. Thus, the gravity model accuracy is improved by over two orders of magnitude.
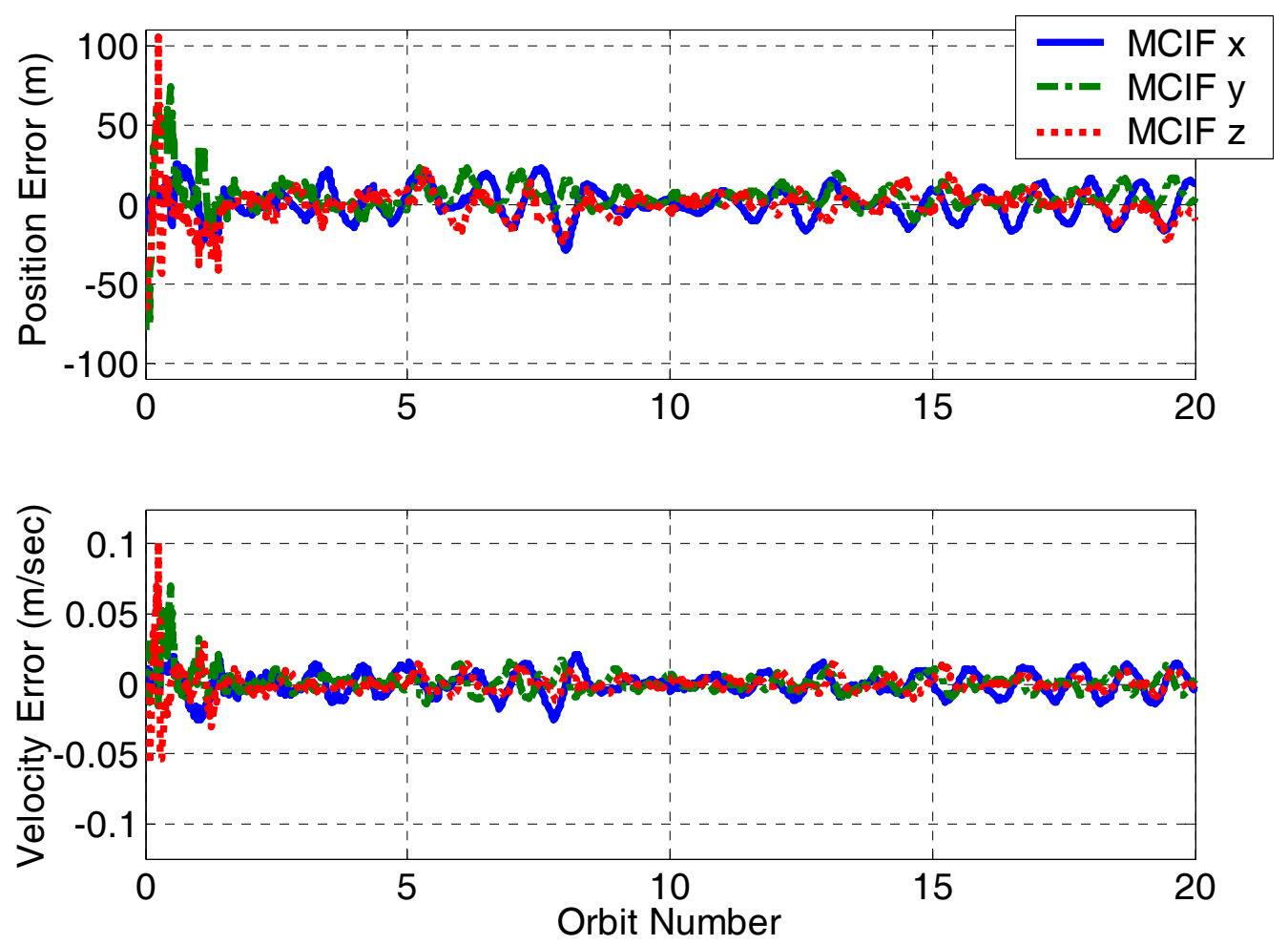

Figure 2. Position and velocity estimation errors for Satellite 1 with perfect a priori knowledge of $\mu$.

The system works less well when there is significant initial uncertainty in $\mu$ if Doppler shift measurements from an Earth-based tracking station are not used. Figure 3 illustrates what happens when the initial uncertainty of $\mu$ is 52 parts in $10^{6}$. The position and velocity estimation errors in Fig. 3 do not reach RMS values as low as those of Fig. 2 after 20 orbits. Even after a month of filtering the per-axis RMS position errors are $17 \mathrm{~m}$, and the per-axis RMS velocity errors are $0.014 \mathrm{~m} / \mathrm{s}$. Similarly, the estimated corrections to the gravitational model are significantly degraded. After one month of filtering, the per-axis RMS acceleration errors are only reduced to $3.7 \times 10^{-5} \mathrm{~m} / \mathrm{s}^{2}$.

The poor performance of the latter case can be attributed to a significant residual error in its estimate of $\mu$. The $\mu$ 
estimate settles to a nearly steady value after about 25 orbits, and this value is in error by 46 parts in $10^{6}$. The $\mu$ estimate improves only very slowly after that, presumably because of its weak observability. If one compares the estimates of the other gravity parameters, $C_{20}$ through $S_{N N}$, between the two cases, then one finds that most of these quantities' estimates are nearly identical. If one analyzes the large position errors on the top plot of Fig. 3 , one finds that these time histories are dominated by a $29 \mathrm{~m}$ altitude bias. This bias is not apparent on the figure because the projection of altitude onto the three axes of the Moon-Centered Inertially-Fixed (MCIF) coordinate system varies with time. The altitude bias is precisely the value that is required to counteract the effect on mean orbital motion of the nearly steady error in $\mu$. This makes sense because the mean rates of the two orbits should be observable from observations of the bearing vector $\Delta \hat{r}_{12}$.
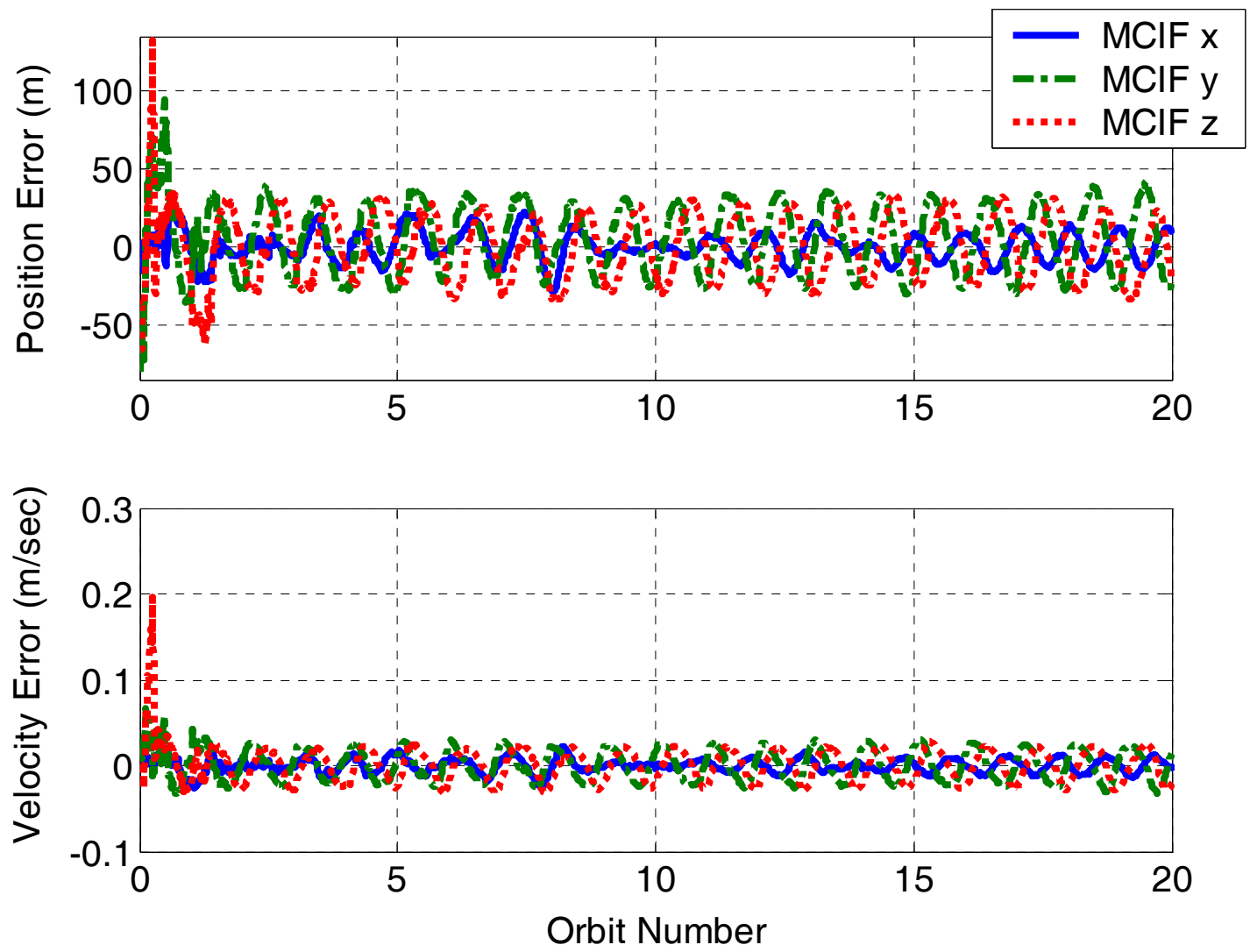

Figure 3. The effect of imperfect a priori knowledge of $\mu$ on the position and velocity estimation errors for Satellite 1.

The correlation of the $\mu$ error with an altitude error and the absence of an error in the mean orbital rates suggests a method of improving the system: Use an Earth-based tracking system in order to make an external measurement of a component of the velocity of one of the spacecraft. If this component is nearly aligned with the actual velocity, then the tracking system will be able to correct the $\mu$ and altitude estimation errors because the inaccurate altitude and the accurate average orbital rate translate into an inaccurate average speed that an Earth-based Doppler shift measurement can detect.

A test has been made of this hypothesis about how to improve the system when $\mu$ is uncertain. The filtering run that produced the poorer results in Fig. 3 has been modified to add a single Doppler shift measurement from an Earth-based tracking station at the very end of the month-long data batch. This addition involves a single measurement update using the velocity component measurement in Eq. (26) with a measurement accuracy of $\sigma_{v 2}=$ $7 \times 10^{-5} \mathrm{~m} / \mathrm{s}$, which is reasonable for a $100 \mathrm{sec}$ tracking run with a carrier phase measurement accuracy of $0.005 \mathrm{~m}$. The resulting improvement in estimation accuracy is dramatic: The $\mu$ estimation error drops by more than 2 orders of magnitude to 0.2 parts in $10^{6}$, the $29 \mathrm{~m}$ altitude bias virtually disappears, and the per-axis RMS gravity modeling 
errors along the two orbits also drop by more than 2 orders of magnitude to $3.2 \times 10^{-7} \mathrm{~m} / \mathrm{s}^{2}$.

\section{Summary and Conclusions}

A proposed method of autonomous orbit determination for a pair of satellites has been extended to include the estimation of corrections to the gravity model of the primary attracting body. The system's EKF operates on relative position measurements between the two satellites in order to estimate the absolute positions and velocities of both spacecraft along with corrections to the central gravitational parameter $\mu$ and the coefficients in a spherical harmonic expansion of the gravity field. The relative range measurements could be made using a radio cross link, and the relative bearing measurements could be made with an optical system, perhaps a modified star camera.

An observability analysis has been performed for a simple version of the problem that involves only a central $1 / r^{2}$ gravitational force, and the system has been found to be observable in cases where the two orbits' altitude time histories are not identical. This result has been confirmed in a numerical study of a more complicated scenario that involves simultaneous estimation of sensor biases, acceleration error terms, and higher-order coefficients in the spherical harmonic gravity model.

Kalman filtering results of simulated truth-model data from a Lunar-orbiting case confirm the system's ability to estimate the orbits and gravity model corrections simultaneously. If the filter starts with a high degree of certainty in $\mu$ and if it processes a month's worth of data, then the RMS accuracies that can be achieved using reasonable sensor technology are on the order of $3.5 \mathrm{~m}$ in absolute position, $0.003 \mathrm{~m} / \mathrm{s}$ in absolute velocity, and $3 \times 10^{-7} \mathrm{~m} / \mathrm{s}^{2}$ in the gravity model. If the initial $\mu$ uncertainty is on the order of 50 parts in $10^{6}$, then the position and velocity accuracies achievable by the filter can degrade by almost an order of magnitude, and the gravity model accuracy can degrade by 2 orders of magnitude. If, however, the system has access to Doppler shift data from an Earth-based tracking system as infrequently as once per month, then the system accuracy can be improved almost to the level that is achievable with perfect a priori knowledge of $\mu$.

The excellent performance of this system and its autonomy or near autonomy make it a good candidate for the estimation of gravity models of remote celestial bodies. In the near term, such a system should be considered for use in geodetic surveys of the Moon and Mars.

\section{Acknowledgment}

The author thanks Dr. F. Landis Markley of the NASA Goddard Space Flight Center for his helpful comments and feedback regarding the initial observability analysis of Section II.

\section{References}

${ }^{1}$ Tapley, B.D., Schutz. B.E., and Born, G.H., Statistical Orbit Determination, Elsevier, (New York, 2004), pp. 93-149, 159261.

${ }^{2}$ Wagner, C.A., Lerch, F.J., Brownd J.E., and Richardson, J.A., "Improvement in Geopotential Derived from Satellite and Surface Data (GEM-7 and GEM-8)," Journal of Geophysical Research, Vol. 82, No. 5, 1977, pp. 901-914.

${ }^{3}$ Tapley, B., Ries, J., Bettadpur, S., Chambers, D., Cheng, M., Condi, F., Gunter, B., Kang, Z., Nagel, P., Pastor, R., Pekker, T., Poole, S., and Wang, F., "GGM02 - An Improved Earth Gravity Field Model from GRACE," Journal of Geodesy, Vol. 79, No. 8, 2005, pp. 467-478.

${ }^{4}$ Anon. "SELENE: The Moon," available on-line at http://www.isas.ac.jp/e/enterp/missions/ selene/index.shtml, Jan. 2007.

${ }^{5}$ Keller, J., "The Lunar Reconnaissance Orbiter -- Instrument Suite and Measurements," available on-line at http://lunar.gsfc.nasa.gov/library/keller51906.pdf, Jan. 2007.

${ }^{6}$ Hupp, E., and Brown, D., "NASA Announces Discover Program Selections," available on-line at http://stardust.jpl.nasa.gov/news/status/061030.html, Oct. 2006.

${ }^{7}$ Anon., "About the Deep Space Network," available on-line at http://deepspace.jpl.nasa.gov/ dsn/, Jan. 2007.

${ }^{8}$ Markley, F.L., "Autonomous Navigation Using Landmark and Intersatellite Data," AIAA Paper No. 84-1987, AIAA/AAS Astrodynamics Conf., Seattle, Washington, Aug. 20-22, 1984.

${ }^{9}$ Psiaki, M.L., “Autonomous Orbit Determination for Two Spacecraft from Relative Position Measurements,” Journal of Guidance, Control, and Dynamics, Vol. 22, No. 2, March-April 1999, pp. 305-312.

${ }^{10}$ Lundberg, J.B., and Schutz, B.E., "Recursion Formulas for Legendre Functions for Use with Nonsingular Geopotential Models," Journal of Guidance, Control, and Dynamics, Vol. 11, No. 1, Jan.-Feb. 1988, pp. 31-38.

${ }^{11}$ Bierman, G.J., Factorization Methods for Discrete Sequential Estimation, Academic Press, (New York, 1977), pp. 69-76, 115-122.

${ }^{12}$ Gill, P.E., Murray, W., and Wright, M.H., Practical Optimization, Academic Press, (New York, 1981), pp. 37-40. 